\title{
In-vivo cardiac DTI: An initial comparison of M012 compensated spin-echo and STEAM
}

\author{
Andrew D Scott ${ }^{1,2^{*}}$, Sonia Nielles-Vallespin ${ }^{3,1}$, Pedro Ferreira ${ }^{1,2}$, Zohya Khalique ${ }^{1}$, Laura-Ann McGill ${ }^{1,2}$, \\ Philip J Kilner ${ }^{1,2}$, Dudley J Pennell ${ }^{1,2}$, David Firmin ${ }^{1,2}$ \\ From 19th Annual SCMR Scientific Sessions \\ Los Angeles, CA, USA. 27-30 January 2016
}

\section{Background}

In-vivo cardiac diffusion tensor imaging (cDTI) has been performed using a stimulated echo (STEAM) sequence for 20 years [1]. While short diffusion gradients make it motion insensitive, it is strain sensitive and SNR inefficient. Recently a spin-echo (SE) sequence with velocity and acceleration compensated diffusion gradients was demonstrated in rats [2] and healthy volunteers using high performance gradients [3]. This sequence is insensitive to strain and should have higher SNR than STEAM, but diffusion gradient duration and hence TE is increased while mixing time is decreased. Here we implement a velocity and acceleration compensated SE cDTI sequence on a clinical 3T scanner and show initial comparisons with STEAM.

\section{Methods}

A SE EPI CDTI sequence was implemented with $0^{\text {th }}, 1^{\text {st }}$ and $2^{\text {nd }}$ order motion-compensated diffusion gradients (M012) [2,3]. Mid-ventricular short-axis cDTI was performed in 10 healthy volunteers on a 3T Siemens Skyra (Gradients $45 \mathrm{mT} / \mathrm{m} @ 200 \mathrm{Tm} / \mathrm{s}$ per axis) with both M012 and STEAM [4]. Acquisitions were performed at end-systole, end-diastole and $150 \mathrm{~ms}$ from the R-wave (average systolic sweet-spot [5]). Time from R-wave to diffusion encoding was matched between sequences. M012 acquisitions used $b_{\text {main }}=450 \mathrm{smm}^{-2}, T E=73 \mathrm{~ms}$ and water-selective excitation. STEAM acquisitions used $\mathrm{b}_{\text {main }}=800 \mathrm{smm}^{-2}, \mathrm{TE}=23 \mathrm{~ms}$ and fat saturation. Both acquisitions used 6 diffusion directions, $b_{\text {ref }}=150 \mathrm{smm}^{-2}$, 6 averages, TR $=2 R R$-intervals, reduced phase field-ofview, $360 \times 135 \times 8 \mathrm{~mm}^{3}$ at $2.8 \times 2.8 \mathrm{~mm}^{2}$ resolution, SENSE $\mathrm{x} 2$ and an identical EPI echo train. Each

Cardiovascular Biomedical Research Unit, The Royal Brompton Hospital,

London, United Kingdom

Full list of author information is available at the end of the article breath-hold was 20RR for both sequences. Since STEAM requires $2 R R$ for diffusion encoding the M012-SE sequence was triggered to alternate $\mathrm{R}$-waves.

\section{Results}

Figure 1 shows parameter maps from one subject using both sequences at all 3 time points. All STEAM acquisitions were considered evaluable. For M012: 1/10 systolic, $3 / 10$ sweet spot and $3 / 10$ diastolic data sets were not evaluable due to bulk motion related signal loss. Figure 2 compares helical angle gradient, absolute second eigenvector angle (E2A) [6], mean diffusivity (MD), fractional anisotropy (FA) and SNR measured in the left ventricle from all acquisitions. MD is lower and FA is higher using M012 (both $\mathrm{p}<0.05$ ). Differences in E2A between systole and diastole are reduced using M012. SNR is higher using STEAM (diastole: $\mathrm{p}<0.05$ ).

\section{Conclusions}

M012 compensated SE cDTI can be performed on most subjects at $3 \mathrm{~T}$ with clinical gradients but its accuracy and reproducibility relative to other techniques requires further evaluation. Preliminary results show that STEAM is more reliable and the expected improvement in SNR using the M012 sequence was not observed. Parameters may vary significantly between techniques due several factors including: T1 and T2-weighting, strain sensitivity, motion sensitivity and mixing time.

\section{Authors' details}

${ }^{1}$ Cardiovascular Biomedical Research Unit, The Royal Brompton Hospital, London, United Kingdom. ${ }^{2}$ National Heart and Lung Institute, Imperial College London, London, United Kingdom. ${ }^{3}$ National Heart Lung and Blood Institute, National Institutes for Health, Bethesda, MD, USA. 


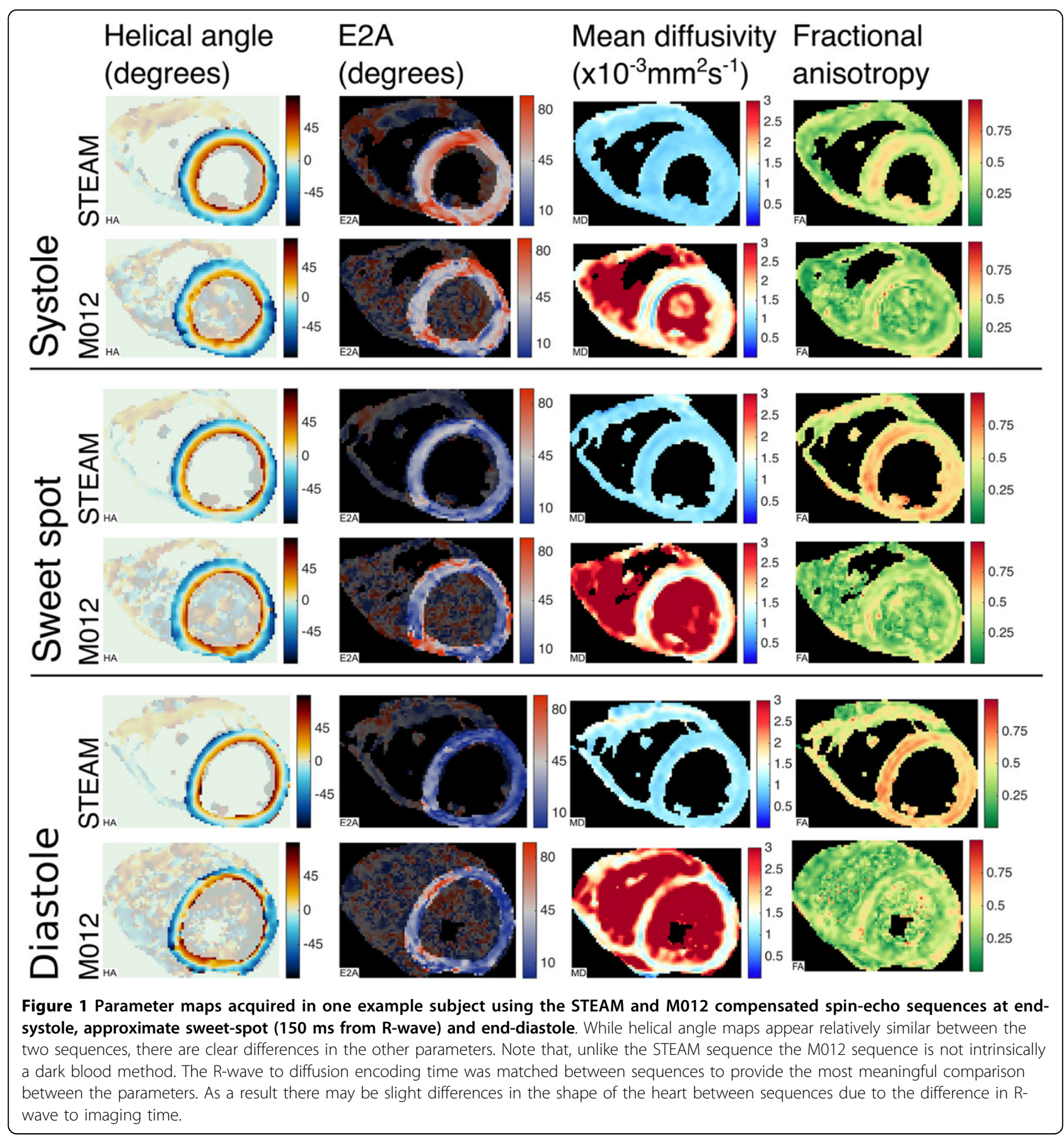

Published: 27 January 2016

\section{References}

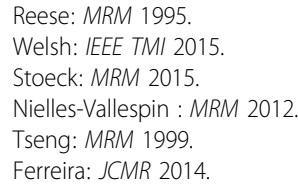

doi:10.1186/1532-429X-18-S1-W19

Cite this article as: Scott et al:: In-vivo cardiac DTI: An initial comparison of M012 compensated spin-echo and STEAM. Journal of Cardiovascular Magnetic Resonance 2016 18(Suppl 1):W19. 


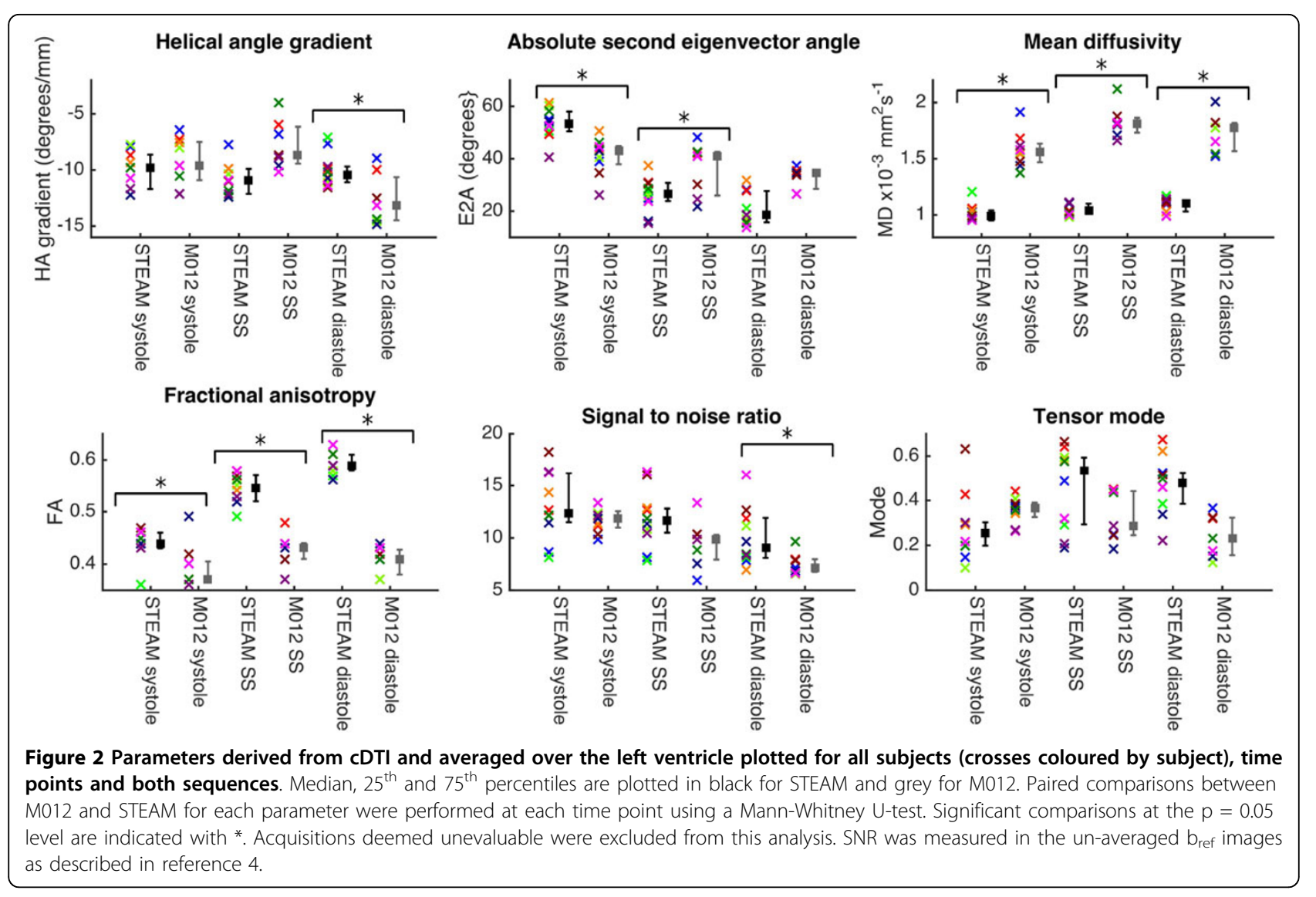

